\title{
CHOI-DAVIS-JENSEN'S INEQUALITY WITHOUT CONVEXITY
}

\section{JADRANKA Mićić, Hamid ReZa Moradi And Shigeru Furuichi}

Abstract. We give the Choi-Davis-Jensen type inequality without using convexity. Applying our main results, we also give new inequalities improving previous known results. In particular, we show some inequalities for relative operator entropies and quantum mechanical entropies.

Mathematics subject classification (2010): Primary 47A63, Secondary 47A64, 26D15, 94A17, 15A39. Keywords and phrases: Choi-Davis-Jensen's inequality, positive linear maps, convex function, operator inequality, Kantorovich inequality.

\section{REFERENCES}

[1] R. Bhatia, C. Davis, A key inequality for functions of matrices, Linear Algebra Appl. 323 (1-3) (2001), 1-5.

[2] R. Bhatia, Positive definite matrices, Princeton University Press, 2007.

[3] M. D. ChoI, A Schwarz inequality for positive linear maps on $C^{*}$-algebras, Illinois J. Math. 18 (1974), 565-574.

[4] Ch. Davis, A Schwarz inequality for convex operator functions, Proc. Amer. Math. Soc. 8 (1957), $42-44$.

[5] S. S. DRAGOMiR, On New Refinements and Reverses of Young's Operator Inequality, arXiv:1510.01314, (2015).

[6] A. Ebadian, I. Nikoufar, M. Eshagi Gordji, Perspectives of matrix convex functions, Proc. Natl. Acad. Sci. USA, 108 (18) (2011), 7313-7314.

[7] E. G. EfFros, A matrix convexity approach to some celebrated quantum inequalities, Proc. Natl. Acad. Sci. USA, 106 (2009), 1006-1008.

[8] J. I. Fujil, M. FujiI, Y. Seo, An extension of the Kubo-Ando theory: Solidarities, Math. Japonica. 35 (1990), 387-396.

[9] T. Furuta, J. Mićić Hot, J. PeČARIĆ And Y. SeO, Mond-Pečarić method in operator inequalities, Element, Zagreb, 2005.

[10] A. W. Marshall, I. OlKin, Matrix versions of Cauchy and Kantorovich inequalities, Aequationes Math. 40 (1990), 89-93.

[11] J. MIĆIĆ, Z. PAVIĆ, J. PEČARIĆ, Jensen's inequality for operators without operator convexity, Linear Algebra Appl. 434 (5) (2011), 1228-1237.

[12] M. ОhYA, D. Petz, Quantum entropy and its use, Springer, Second Edition 2004.

[13] S. Furuichi, K. YAnagi, K. Kuriyama, Fundamental properties of Tsallis relative entropy, J. Math. Phys. 45 (2004), 4868-4876.

[14] S. Furuichi, Matrix trace inequalities on the Tsallis entropies, J. Inequal. Pure Appl. Math. 9, Art. 1 (2008), 1-7.

[15] J. I. FUJiI, E. KAMEI, Relative operator entropy in non-commutative information theory, Math. Japon. 34 (1989), 341-348.

[16] J. MiĆIĆ, J. PeČARIĆ, Y. SEO, M. TominagA, Inequalities for positive linear maps on Hermitian matrices, Math. Inequal. Appl. 3 (2000), 559-591.

[17] K. YAnAGi, K. KURIYAma, S. FurUichi, Generalized Shannon inequalities based on Tsallis relative operator entropy, Linear Algebra Appl. 394 (2005), 109-118. 